Mathematical Modelling and AnAlysis

Volume 16 Number 3, September 2011, 401-417

Doi:10.3846/13926292.2011.602125

(C) Vilnius Gediminas Technical University, 2011
Publisher: Taylor\&Francis and VGTU

www.tandfonline.com/loi/tmma20

Online ISSN: $1648-3510$

Print ISSN: 1392-6292

\title{
Linear Differential Equation with Additional Conditions and Formulae for Green's Function*
}

\section{Svetlana Roman}

\author{
Vilnius University \\ Akademijos 4, Vilnius, LT-08663, Lithuania \\ E-mail(corresp.): svetlana.roman@mii.vu.lt \\ Received June 16, 2011; revised July 1, 2011; published online August 1, 2011
}

\begin{abstract}
In this paper, we investigate the $m$-order linear ordinary differential equation with $m$ linearly independent additional conditions. We have found the solution to this problem and give the formula and the existence condition of Green's function. We compare two Green's functions for two such problems with different additional conditions and apply these results to the problems with nonlocal boundary conditions.
\end{abstract}

Keywords: Linear ODE, Green's function, Nonlocal Boundary Conditions.

AMS Subject Classification: 34B05; 34B10; 34B27.

\section{Introduction}

Green's functions play an important role in the theory of linear ODE and PDE. Their exact form depends on the differential equation and the type of boundary conditions (BC). Green's function helps us investigate the existence and uniqueness of the solutions for many boundary-value problems (BVP) [23]. For multidimensional stationary problems and non-stationary problems the formulae for Green's function are more complicated and Green's functions are represented as functional series even for simple rectangular, spherical and cylindrical domains [15].

The formulae of Green's functions for many problems with classical BCs are presented in [4]. In this book, Green's functions are constructed for regular and singular BVP for ODEs, the Helmholtz equation and linear non-stationary equations (heat equation, wave equation). We can reduce the Sturm-Liouville problem with classical boundary conditions to an integral equation with a symmetrical continuous kernel, which is Green's function for the Sturm-Liouville differential operator. The investigation of Green's functions for problems with nonclassical BCs is quite a new area. We often have no self-adjoint operators

* This research was funded by a grant (No. MIP-051/2011) from the Research Council of Lithuania. 
in this case. Problems with Nonlocal Boundary Conditions (NBC) $[13,14,20$, $21,22,24,27]$ are good examples in this area.

Green's functions for second-order BVPs with various NBCs have been constructed in [32]. In [17, 26], Green's function has been constructed for a secondorder and third-order linear ODE with two and three additional conditions, respectively. Green's function for discrete second-order problems with NBCs was investigated in [19]. The examples, in which the expression of Green's function obtained in [26] is used, are presented in [16, 18]. Third- and higher-order BVPs have been considered in many papers. In $[28,31]$, the authors investigate the existence of positive solutions to the third-order BVPs, they apply various methods: lower and upper solution method, and Leggett-Williams fixed-point theorem, Leray-Schauder continuation principle. In these references, expressions and some useful properties of Green's functions have been obtained. The uniqueness and the necessary and sufficient conditions for the existence of solutions to the fourth-order problems with various BCs have been investigated in $[12,30]$.

Xie et al. [29] have studied the existence of positive solutions, they have presented Green's function for the $m$-order three-point BVP

$$
\begin{aligned}
& u^{(m)}(t)+h(t) f(t, u(t))=0, \quad t \in[a, b], \\
& u(a)=\alpha u(\nu), u^{\prime}(a)=0, \ldots, u^{(m-2)}(a)=0, u(b)=\beta u(\nu) .
\end{aligned}
$$

This problem is also investigated by Ji and Guo [7] for $a=0$ and Hao et al. [6] for $a=\alpha=0$ and $b=1$. Jiang [8] has investigated a similar problem with $n$-point BCs

$$
u(0)=u^{\prime}(0)=\cdots=u^{(m-2)}(0)=\theta, \quad u(1)=\sum_{i=1}^{n-2} k_{i} u\left(\xi_{i}\right) .
$$

The problem for $\theta=0$ with the latter condition replaced with $\alpha u^{(m-2)}(\nu)=$ $u^{(m-2)}(1)$ is investigated by Graef et al. [5]. The main objective of these papers is to investigate the existence and multiplicity of solutions. To this end, the authors have constructed Green's functions and studied their properties.

In this paper, we consider the $m$-order linear ODE

$$
L u:=u^{(m)}+a^{m-1}(x) u^{(m-1)}+\cdots+a^{1}(x) u^{\prime}+a^{0}(x) u=f .
$$

Expressions for Green's functions have been obtained using the method of variation of parameters [3]. The advantage of this method is that it is possible to construct Green's function for a nonhomogeneous equation (1.1) with the variable coefficients $a^{0}, \ldots, a^{m-1} \in C[0, L]$ and various additional conditions (for example NBCs).

The structure of the paper is as follows. In Section 2, we review the properties of functional determinants and linear functionals. We give a definition of the Generalized Fundamental Solution in Section 3. Then we construct a special basis in Section 4 and find an expression for the solution of the $m$-order linear ODE with $m$ additional conditions in Section 5. Formulae for Green's functions are derived in Section 6. Finally, in Section 7, we apply formulae 
to Green's functions for problems with NBCs and propose examples how to construct Green's function for nonclassical problems. The main result of this article is formulated in Theorems 1 and 2 .

\section{Notation}

Let $F=F(X):=\{u \mid u: X \rightarrow \mathbb{K}\}$ be a linear space of real (complex) functions, where $X$ can be any set, $\mathbb{K}=\mathbb{R}$ or $\mathbb{K}=\mathbb{C}$ and $1 \leqslant k \in \mathbb{N}$. If we have a vector $\boldsymbol{x}=\left(x_{1}, \ldots, x_{k}\right) \in X^{k}$ and the vector-function $\boldsymbol{w}=\left[w^{1}, \ldots, w^{k}\right] \in F^{k}$, then we consider the matrix $[\boldsymbol{w}](\boldsymbol{x}) \in M_{k \times k}(\mathbb{K})$ and its determinant:

$$
\begin{gathered}
{[\boldsymbol{w}](\boldsymbol{x})=\left[w^{1}, \ldots, w^{k}\right]\left(x_{1}, \ldots, x_{k}\right):=\left(\begin{array}{ccc}
w^{1}\left(x_{1}\right) & \ldots & w^{1}\left(x_{k}\right) \\
\ldots & \ldots & \ldots \\
w^{k}\left(x_{1}\right) & \ldots & w^{k}\left(x_{k}\right)
\end{array}\right),} \\
D[\boldsymbol{w}](\boldsymbol{x})=\operatorname{det}\left[w^{1}, \ldots, w^{k}\right]\left(x_{1}, \ldots, x_{k}\right):=\left|\begin{array}{ccc}
w^{1}\left(x_{1}\right) & \ldots & w^{1}\left(x_{k}\right) \\
\ldots & \ldots & \ldots \\
w^{k}\left(x_{1}\right) & \ldots & w^{k}\left(x_{k}\right)
\end{array}\right| .
\end{gathered}
$$

We consider the space $F^{*}$ of linear functionals in the space $F$, and we use the notation $\langle f, w\rangle,\langle f(\cdot), w(\cdot)\rangle,\langle f(x), w(x)\rangle$ for a functional $f$ value of the function $w$. For example, we have a regular functional $f \in\left(C^{p}[0, L]\right)^{*}$ if there exists $\tilde{f} \in L^{1}(0, L)$ such that $\langle f, w\rangle=(\tilde{f}, w):=\int_{0}^{L} \tilde{f}(x) w(x) d x$, and $\delta_{x}^{(r)}$ is a singular functional such that $\left\langle\delta_{x}^{(r)}, w\right\rangle=(-1)^{r} w^{(r)}(x), r=\overline{0, p}$. We denote $\delta_{x}:=\delta_{x}^{(0)}$. If we have the vector-function $\boldsymbol{w}$ and the vector-functional $\boldsymbol{f}=\left(f_{1}, \ldots, f_{k}\right) \in\left(F^{*}\right)^{k}$, then we define the matrix $(\boldsymbol{f})[\boldsymbol{w}]$ and its determinant:

$$
\begin{gathered}
(\boldsymbol{f})[\boldsymbol{w}]=\left(f_{1}, \ldots, f_{k}\right)\left[w^{1}, \ldots, w^{k}\right]:=\left(\begin{array}{ccc}
\left\langle f_{1}, w^{1}\right\rangle & \ldots & \left\langle f_{k}, w^{1}\right\rangle \\
\ldots & \ldots & \ldots \\
\left\langle f_{1}, w^{k}\right\rangle & \ldots & \left\langle f_{k}, w^{k}\right\rangle
\end{array}\right), \\
D(\boldsymbol{f})[\boldsymbol{w}]=\operatorname{det}\left(f_{1}, \ldots, f_{k}\right)\left[w^{1}, \ldots, w^{k}\right]:=\left|\begin{array}{ccc}
\left\langle f_{1}, w^{1}\right\rangle & \ldots & \left\langle f_{k}, w^{1}\right\rangle \\
\ldots & \ldots & \ldots \\
\left\langle f_{1}, w^{k}\right\rangle & \ldots & \left\langle f_{k}, w^{k}\right\rangle
\end{array}\right| .
\end{gathered}
$$

Example 1. The relations between two matrices and two determinants are:

$$
\left(\delta_{x_{1}}, \ldots, \delta_{x_{k}}\right)[\boldsymbol{w}]=[\boldsymbol{w}](\boldsymbol{x}), \quad D\left(\delta_{x_{1}}, \ldots, \delta_{x_{k}}\right)[\boldsymbol{w}]=D[\boldsymbol{w}](\boldsymbol{x}) .
$$

Example 2. We will use functions $\hat{f}^{l}[\boldsymbol{w}] \in F(X), l=\overline{1, k}$ :

$$
\hat{f}^{l}[\boldsymbol{w}](x):=D\left(f_{1}, \ldots, f_{l-1}, \delta_{x}, f_{l+1}, \ldots, f_{k}\right)[\boldsymbol{w}] .
$$

Lemma 1. Let us take vectors $\mathbf{A}_{j}, \mathbf{B}_{j} \in M_{k \times 1}(\mathbb{K}), j=1, \ldots, k$ and matrices $\mathbf{A}=\left(\mathbf{A}_{1}, \ldots, \mathbf{A}_{k}\right), \mathbf{B}=\left(\mathbf{B}_{1}, \ldots, \mathbf{B}_{k}\right) \in M_{k \times k}(\mathbb{K})$. Then the equality

$$
\left|\begin{array}{ccc}
\operatorname{det}\left(\mathbf{B}_{1}, \mathbf{A}_{2}, \ldots, \mathbf{A}_{k}\right) & \ldots & \operatorname{det}\left(\mathbf{B}_{k}, \mathbf{A}_{2}, \ldots, \mathbf{A}_{k}\right) \\
\operatorname{det}\left(\mathbf{A}_{1}, \mathbf{B}_{1}, \ldots, \mathbf{A}_{k}\right) & \ldots & \operatorname{det}\left(\mathbf{A}_{1}, \mathbf{B}_{k}, \ldots, \mathbf{A}_{k}\right) \\
\ldots & \ldots & \ldots \\
\operatorname{det}\left(\mathbf{A}_{1}, \mathbf{A}_{2}, \ldots, \mathbf{B}_{1}\right) & \ldots & \operatorname{det}\left(\mathbf{A}_{1}, \mathbf{A}_{2}, \ldots, \mathbf{B}_{k}\right)
\end{array}\right|=\operatorname{det} \mathbf{B} \cdot(\operatorname{det} \mathbf{A})^{k-1}
$$


is valid.

Proof. An adjugate of $\mathbf{A}$ is the matrix adj $\mathbf{A} \in M_{k \times k}(\mathbb{K})$, whose $(i, j)$ entry is the $(j, i)$ cofactor of $\mathbf{A}$ (see [11]):

$$
\begin{aligned}
\operatorname{adj} \mathbf{A} & =\left(\begin{array}{ccc}
A_{1}^{1} & \ldots & A_{k}^{1} \\
\ldots & \ldots & \ldots \\
A_{1}^{k} & \ldots & A_{k}^{k}
\end{array}\right)^{\mathrm{T}} \\
& =\left(\begin{array}{ccc}
\operatorname{det}\left(\mathbf{E}_{1}, \mathbf{A}_{2}, \ldots, \mathbf{A}_{k}\right) & \ldots & \operatorname{det}\left(\mathbf{E}_{k}, \mathbf{A}_{2}, \ldots, \mathbf{A}_{k}\right) \\
\operatorname{det}\left(\mathbf{A}_{1}, \mathbf{E}_{1}, \ldots, \mathbf{A}_{k}\right) & \ldots & \operatorname{det}\left(\mathbf{A}_{1}, \mathbf{E}_{k}, \ldots, \mathbf{A}_{k}\right) \\
\ldots & \ldots & \ldots \\
\operatorname{det}\left(\mathbf{A}_{1}, \mathbf{A}_{2}, \ldots, \mathbf{E}_{1}\right) & \ldots & \operatorname{det}\left(\mathbf{A}_{1}, \mathbf{A}_{2}, \ldots, \mathbf{E}_{k}\right)
\end{array}\right)
\end{aligned}
$$

The adjugate of $\mathbf{A}$ has the property $\operatorname{det}(\operatorname{adj} \mathbf{A})=(\operatorname{det} \mathbf{A})^{k-1}(\operatorname{see}[11])$. So, we derive the equality

$$
\left|\begin{array}{ccc}
\operatorname{det}\left(\mathbf{E}_{1}, \mathbf{A}_{2}, \ldots, \mathbf{A}_{k}\right) & \ldots & \operatorname{det}\left(\mathbf{E}_{k}, \mathbf{A}_{2}, \ldots, \mathbf{A}_{k}\right) \\
\operatorname{det}\left(\mathbf{A}_{1}, \mathbf{E}_{1}, \ldots, \mathbf{A}_{k}\right) & \ldots & \operatorname{det}\left(\mathbf{A}_{1}, \mathbf{E}_{k}, \ldots, \mathbf{A}_{k}\right) \\
\ldots & \ldots & \ldots \\
\operatorname{det}\left(\mathbf{A}_{1}, \mathbf{A}_{2}, \ldots, \mathbf{E}_{1}\right) & \ldots & \operatorname{det}\left(\mathbf{A}_{1}, \mathbf{A}_{2}, \ldots, \mathbf{E}_{k}\right)
\end{array}\right|=(\operatorname{det} \mathbf{A})^{k-1}
$$

The function $\mathcal{D}: \mathbb{K}^{k} \times \cdots \times \mathbb{K}^{k} \rightarrow \mathbb{K}$ (for fixed vectors $\mathbf{A}_{1}, \ldots, \mathbf{A}_{k}$ )

$$
\mathcal{D}\left(\mathbf{B}_{1}, \ldots, \mathbf{B}_{k}\right):=\left|\begin{array}{ccc}
\operatorname{det}\left(\mathbf{B}_{1}, \mathbf{A}_{2}, \ldots, \mathbf{A}_{k}\right) & \ldots & \operatorname{det}\left(\mathbf{B}_{k}, \mathbf{A}_{2}, \ldots, \mathbf{A}_{k}\right) \\
\operatorname{det}\left(\mathbf{A}_{1}, \mathbf{B}_{1}, \ldots, \mathbf{A}_{k}\right) & \ldots & \operatorname{det}\left(\mathbf{A}_{1}, \mathbf{B}_{k}, \ldots, \mathbf{A}_{k}\right) \\
\ldots & \ldots & \ldots \\
\operatorname{det}\left(\mathbf{A}_{1}, \mathbf{A}_{2}, \ldots, \mathbf{B}_{1}\right) & \ldots & \operatorname{det}\left(\mathbf{A}_{1}, \mathbf{A}_{2}, \ldots, \mathbf{B}_{k}\right)
\end{array}\right|
$$

is an alternating multilinear function. Therefore, the equality $\mathcal{D}\left(\mathbf{B}_{1}, \ldots, \mathbf{B}_{k}\right)=$ $\operatorname{det} \mathbf{B} \cdot \mathcal{D}\left(\mathbf{E}_{1}, \ldots, \mathbf{E}_{k}\right)$ is valid. But $\mathcal{D}\left(\mathbf{E}_{1}, \ldots, \mathbf{E}_{k}\right)$ is equal to the left-hand side of (2.2). So, the lemma is proved.

Corollary 1. The following equality is valid:

$$
D(\boldsymbol{g})[\hat{\boldsymbol{f}}[\boldsymbol{w}]]=D(\boldsymbol{g})[\boldsymbol{w}] \cdot(D(\boldsymbol{f})[\boldsymbol{w}])^{k-1},
$$

where $\hat{f}$ is defined by formula $(2.1)$.

Wronskian $W[\boldsymbol{w}](x)$ and determinant $\widetilde{W}[\boldsymbol{w}](x, y)$ are used in the theory of linear differential equations for $\boldsymbol{w}=\left[w^{1}, \ldots, w^{k}\right] \in C^{k-1}[0, L]$ (see [3]):

$$
\begin{aligned}
& W[\boldsymbol{w}](y):=\left|\begin{array}{ccccc}
w^{1}(y) & \left(w^{1}\right)^{\prime}(y) & \ldots & \left(w^{1}\right)^{(k-2)}(y) & \left(w^{1}\right)^{(k-1)}(y) \\
\ldots & \ldots & \ldots & \ldots & \ldots \\
w^{k}(y) & \left(w^{k}\right)^{\prime}(y) & \ldots & \left(w^{k}\right)^{(k-2)}(y) & \left(w^{k}\right)^{(k-1)}(y)
\end{array}\right|, y \in[0, L], \\
& \widetilde{W}[\boldsymbol{w}](x, y):=\left|\begin{array}{ccccc}
w^{1}(y) & \left(w^{1}\right)^{\prime}(y) & \ldots & \left(w^{1}\right)^{(k-2)}(y) & w^{1}(x) \\
\ldots & \ldots & \ldots & \ldots & \ldots \\
w^{k}(y) & \left(w^{k}\right)^{\prime}(y) & \ldots & \left(w^{k}\right)^{(k-2)}(y) & w^{k}(x)
\end{array}\right|, x, y \in[0, L] .
\end{aligned}
$$


Then we get from equality (2.3):

$$
\begin{aligned}
& W[\hat{\boldsymbol{f}}[\boldsymbol{w}]](y)=W[\boldsymbol{w}](y) \cdot(D(\boldsymbol{f})[\boldsymbol{w}])^{k-1} \\
& \widetilde{W}[\hat{\boldsymbol{f}}[\boldsymbol{w}]](x, y)=\widetilde{W}[\boldsymbol{w}](x, y) \cdot(D(\boldsymbol{f})[\boldsymbol{w}])^{k-1}
\end{aligned}
$$

For $W[\boldsymbol{w}](y) \neq 0$, let us introduce $V[\boldsymbol{w}](x, y):=\widetilde{W}[\boldsymbol{w}](x, y) / W[\boldsymbol{w}](y)$. The function $V[\boldsymbol{w}](x, y)$ is invariant with respect to $\left\{w^{1}, \ldots, w^{k}\right\}$. If $\boldsymbol{w} \in C^{k-1+l}[0, L]$, $l=\overline{0, k-1}$, then $\frac{\partial^{k-1+2 l}}{\partial x^{k-1+l} \partial y^{l}} V \in C[0, L]^{2}$, and

$$
\begin{aligned}
& \left.\frac{\partial^{n+l}}{\partial x^{n} \partial y^{l}} V(x, y)\right|_{y=x}=0, \quad n=0, \ldots, k-2-l, \\
& \left.\frac{\partial^{k-1}}{\partial x^{k-1-l} \partial y^{l}} V(x, y)\right|_{y=x}=(-1)^{l} .
\end{aligned}
$$

Let us consider functions $w_{i} \in C^{p}\left[\xi_{i}, \xi_{i+1}\right], i=\overline{0, N-1}, p=0,1, \ldots$, where $-\infty<\xi_{0}=a<\xi_{1}<\cdots<\xi_{N-1}<\xi_{N}=b<+\infty(N \in \mathbb{N})$ and a function $w$, such that $w(x)=w_{i}(x)$ for $x \in\left(\xi_{i}, \xi_{i+1}\right)$. This function is not defined at the points $\xi \in \Xi_{N}=\left\{\xi_{0}, \xi_{1}, \ldots, \xi_{N}\right\}$. We must consider the classes of functions in $[a, b]$ (two functions $w$ and $v$ are equivalent if $w=v$ for all $x \in \cup_{i=0}^{N-1}\left(\xi_{i}, \xi_{i+1}\right)$ ). We can change the $N$ and the set $\Xi_{N}$. We denote a linear space of all such functions (classes) as $C^{[p]}[a, b]$, and $C^{r,[p]}[a, b]:=C^{[p]}[a, b] \cap C^{r}[a, b], 0 \leqslant r \leqslant p$.

Let us recall the Heaviside function

$$
H(x):= \begin{cases}0, & x<0 \\ 1, & x>0\end{cases}
$$

The function $H(x) \in C^{[\infty]}[-a, a]$ with $\xi_{0}=-a, \xi_{1}=0, \xi_{2}=a>0$. In this example, $H \equiv 0$ for $x \in[-a, 0]$ and $H \equiv 1$ for $x \in[0, a]$.

Let us consider the function

$$
G^{c}(x, y):=H(x-y) V(x, y) .
$$

In the domain $[0, L]^{2}$, we consider two parts $D_{y}:=\left\{(x, y) \in[0, L]^{2}: y \geqslant x\right\}$ and $D_{x}:=\left\{(x, y) \in[0, L]^{2}: y \leqslant x\right\}$. The function $G^{c} \equiv 0$ in $D_{y}$ and $G^{c}=V$ in $D_{x}$. If $\boldsymbol{w} \in C^{k-1+l}[0, L], l=\overline{0, k-1}$, then $G^{c} \in C^{l}[0, L]^{2}$ and

$$
\begin{gathered}
\frac{\partial^{n+l} G^{c}(x, y)}{\partial x^{n} \partial y^{l}} \in C[0, L]^{2}, n=\overline{0, k-2-l}, \\
\frac{\partial^{k-1+l} G^{c}(x, x+0)}{\partial x^{k-1} \partial y^{l}}=\frac{\partial^{k-1+l} G^{c}(x-0, x)}{\partial x^{k-1} \partial y^{l}}=0, \\
\frac{\partial^{k-1+l} G^{c}(x, x-0)}{\partial x^{k-1} \partial y^{l}}=\frac{\partial^{k-1+l} G^{c}(x+0, x)}{\partial x^{k-1} \partial y^{l}}=(-1)^{l} .
\end{gathered}
$$

Note, that $G^{c}\left(x, y_{0}\right) \in C_{x}^{k-2,[k-1+l]}[0, L]$ for all $y_{0} \in[0, L]$ with $\xi_{0}=0, \xi_{1}=y_{0}$, $\xi_{2}=L ; G^{c}\left(x_{0}, y\right) \in C_{y}^{l}[0, L], l<k-1, G^{c}\left(x_{0}, y\right) \in C_{y}^{k-2,[k-1]}[0, L], l=k-1$, with $\xi_{0}=0, \xi_{1}=x_{0}, \xi_{2}=L$ for all $x_{0} \in[0, L]$. 


\section{Fundamental Solutions}

Let $\boldsymbol{u}=\left[u^{1}, \ldots, u^{m}\right] \in C^{m}[0, L], m \geqslant 1$, such that $W[\boldsymbol{u}](x) \neq 0$ for all $x \in[0, L]$. These functions are solutions of the $m$-order linear homogeneous differential equation

$$
\mathcal{L} u:=u^{(m)}+a^{m-1} u^{(m-1)}+\cdots+a^{1} u^{\prime}+a^{0} u=0,
$$

where

$$
a^{i}[\boldsymbol{u}](x)=-\frac{\operatorname{det}\left(\boldsymbol{u}(x), \ldots, \boldsymbol{u}^{(i-1)}(x), \boldsymbol{u}^{(m)}(x), \boldsymbol{u}^{(i+1)}(x), \ldots, \boldsymbol{u}^{(m-1)}(x)\right)}{W[\boldsymbol{u}](x)},
$$

$i=\overline{0, m-1}$. Thus, the functions $a^{0}(x), \ldots, a^{m-1}(x) \in C[0, L]$ define all the solutions, i.e., the linear space $S:=\left\{u \in C^{m}[0, L]: \mathcal{L} u=0\right\}$ that can be described by the fundamental system $\left\{u^{1}, \ldots, u^{m}\right\}$, and, conversely, the fundamental system $\left\{u^{1}, \ldots, u^{m}\right\}$ defines $a^{0}(x), \ldots, a^{m-1}(x)$. If $\left\{\bar{u}^{1}, \ldots, \bar{u}^{m}\right\}$ is another fundamental system, and $\left[\bar{u}^{1}, \ldots, \bar{u}^{m}\right]=\mathbf{P}\left[u^{1}, \ldots, u^{m}\right]$, where $\mathbf{P} \in G L_{m}(\mathbb{K})$, then $a^{i}[\overline{\boldsymbol{u}}]=a^{i}[\boldsymbol{u}](x), i=\overline{0, m-1}$, i.e., they are invariant with respect to the fundamental system.

Definition 1 [Fundamental Solution, see [9]]. The function $g(x, y)$, defined on $0 \leq x, y \leq L$, is called the fundamental solution of differential equation (3.1) if it has the following properties:

1. $g(x, y)$ has $m$ partial derivatives in $x$ in each of the two triangles $D_{y}$ and $D_{x}$ and these derivatives are continuous in both $x$ and $y$;

2. $g(x, y)$ (as a function of $x$ ) satisfies equation (3.1) in each of those triangles;

3. $g(x, y)$ is continuous in the whole square $0 \leq x, y \leq L$ and has partial derivatives in $x$ up to order $m-2$ and derivatives are continuous in both $x$ and $y$ in this square;

4. The equality

$$
\frac{\partial^{m-1} g(y+0, y)}{\partial x^{m-1}}-\frac{\partial^{m-1} g(y-0, y)}{\partial x^{m-1}}=1
$$

is valid for $0<y<L$.

The function $G^{c}$ (see (2.6)) is an example of the fundamental solution and its domain is shown in Figure 1a.

Let us consider the finite set $\Xi_{N}=\left\{\xi_{0}, \xi_{1}, \ldots, \xi_{N}\right\}$ where $\xi_{0}=0<\xi_{1}<$ $\cdots<\xi_{N-1}<\xi_{N}=L(N \in \mathbb{N})$. The lines $y=\xi_{i}, i=\overline{0, N}$ as well as $y=x$ divide the square $[0, L]^{2}$ into triangles and trapezoids: $D_{x}^{i}, D_{y}^{i}, i=\overline{0, N-1}$ (see Figure $1 \mathrm{~b}$ ). We use this notation for triangles and trapezoids with boundary, i.e., $D_{x}^{i}, D_{y}^{i}, i=\overline{0, N-1}$ are closed sets.

Definition 2 [Generalized Fundamental Solution]. The function $g(x, y)$ defined on $0 \leq x, y \leq L$ is called the Generalized Fundamental Solution (GFS) of differential equation (3.1) if it has the following properties: 


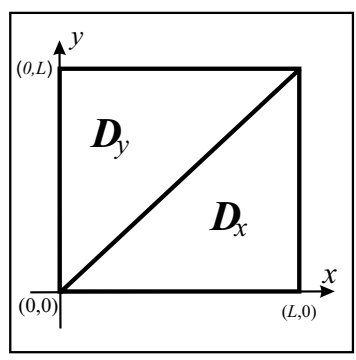

(a) classical fundamental solution

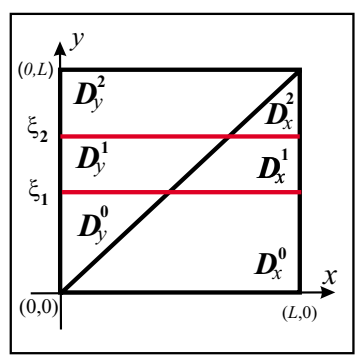

(b) generalized fundamental solution

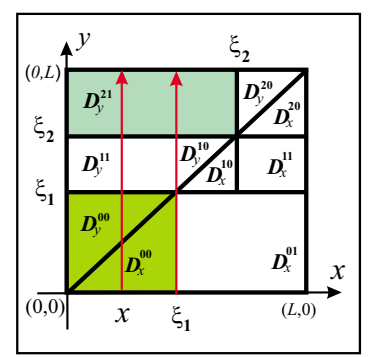

(c)

Figure 1. Domains of the Fundamental Solutions.

1. $g(x, y)$ has $m$ partial derivatives in $x$ in each figure $D_{y}^{i}$ and $D_{x}^{i}, i=$ $\overline{0, N-1}$ and these derivatives are continuous in both $x$ and $y$;

2. $g(x, y)$ (as a function of $x$ ) satisfies equation (3.1) in each of those figures, i.e., $\mathcal{L}_{x} g(x, y)=0$;

3. $g(x, y)$ is continuous in each rectangle $D_{x}^{i} \cup D_{y}^{i}, i=\overline{0, N-1}$ and has partial derivatives in $x$ up to order $m-2$ and derivatives are continuous in both $x$ and $y$ in these rectangles;

4. The equality

$$
\frac{\partial^{m-1} g(y+0, y)}{\partial x^{m-1}}-\frac{\partial^{m-1} g(y-0, y)}{\partial x^{m-1}}=1
$$

is valid for $y \in[0, L] \backslash \Xi_{N}$.

Remark 1. We can rewrite equality (3.2) as

$$
\frac{\partial^{m-1} g(x, x-0)}{\partial x^{m-1}}-\frac{\partial^{m-1} g(x, x+0)}{\partial x^{m-1}}=1, \quad x \in[0, L] \backslash \Xi_{N} .
$$

Remark 2. Each fundamental solution is a generalized fundamental solution $\left(N=1, \xi_{0}=0, \xi_{1}=L\right)$, too. In this paper, we regard the fundamental solution as a Generalized Fundamental Solution.

If $g$ is GFS and $f \in C[0, L]$, then we define the integral

$$
u(x)=\int_{0}^{L} g(x, y) f(y) d y=\sum_{j=1}^{N} \int_{\xi_{j-1}}^{\xi_{j}} g(x, y) f(y) d y .
$$

We have the following formulae

$$
u^{(p)}(x)=\sum_{j=1}^{N} \int_{\xi_{j-1}}^{\xi_{j}} \frac{\partial^{p} g(x, y)}{\partial x^{p}} f(y) d y, \quad p=\overline{1, m-2}
$$


(see the definition of GFS, Property 3), and

$$
\begin{aligned}
u^{(m-1)}(x) & =\sum_{j=1, j \neq k}^{N} \int_{\xi_{j-1}}^{\xi_{j}} \frac{\partial^{m-1} g(x, y)}{\partial x^{m-1}} f(y) d y \\
& +\int_{\xi_{k-1}}^{x} \frac{\partial^{m-1} g(x, y)}{\partial x^{m-1}} f(y) d y+\int_{x}^{\xi_{k}} \frac{\partial^{m-1} g(x, y)}{\partial x^{m-1}} f(y) d y
\end{aligned}
$$

(see the definition of GFS, Property 1), and

$$
\begin{aligned}
u^{(m)}(x) & =\sum_{j=1, j \neq k}^{N} \int_{\xi_{j-1}}^{\xi_{j}} \frac{\partial^{m} g(x, y)}{\partial x^{m}} f(y) d y \\
& +\int_{\xi_{k-1}}^{x} \frac{\partial^{m} g(x, y)}{\partial x^{m}} f(y) d y+\int_{x}^{\xi_{k}} \frac{\partial^{m} g(x, y)}{\partial x^{m}} f(y) d y+f(x)
\end{aligned}
$$

(see the definition of GFS, Properties 1 and 4). Consequently, we get that $u \in C^{m}[0, L]$. We prove that

$$
\begin{aligned}
\mathcal{L} u & =\sum_{j=1, j \neq k}^{N} \int_{\xi_{j-1}}^{\xi_{j}} \mathcal{L}_{x} g(x, y) f(y) d y \\
& +\int_{\xi_{k-1}}^{x} \mathcal{L}_{x} g(x, y) f(y) d y+\int_{x}^{\xi_{k}} \mathcal{L}_{x} g(x, y) f(y) d y+f(x)=f(x)
\end{aligned}
$$

for $x \in\left[\xi_{k-1}, \xi_{k}\right]$ (see the definition of GFS, Property 2). So, $u$ satisfies the equation $\mathcal{L} u=f$.

Remark 3 [see [9]]. If $g(x, y)$ is some GFS, then the formula for all GFS is

$$
g(x, y)+c_{1}(y) u^{1}(x)+\cdots+c_{m}(y) u^{m}(x),
$$

where $\boldsymbol{u}=\left[u^{1}, \ldots, u^{m}\right] \in C^{m}[0, L]$ is the fundamental system of homogeneous equation (3.1) and $c_{j} \in C^{[0]}[0, L]$. For example, we can take

$$
G^{c}(x, y)+c_{1}(y) u^{1}(x)+\cdots+c_{m}(y) u^{m}(x) .
$$

Remark 4. GFS has the properties of fundamental solutions in some subdomains of $[0, L]^{2}$ such as $D_{y}^{00} \cup D_{y}^{00}$ and the properties of the solution to the homogeneous problem in some subdomains of $[0, L]^{2}$ such as $D_{y}^{20}$ (see Figure 1c).

Let us consider linear integral operators $A_{j}: C[0, L] \rightarrow C[0, L]$ :

$$
\left(A_{j} u\right)(x)=\int_{0}^{L} \frac{\partial^{j} g(x, y)}{\partial x^{j}} u(y) d y+u(x) \delta_{j m}, \quad j=0, \ldots, m .
$$

Lemma 2. Integral linear operators $A_{j}, j=\overline{0, m}$, are completely continuous operators.

Proof. It is easy to see that the kernels $K_{j}(x, y)=\frac{\partial^{j} g(x, y)}{\partial x^{j}}$ satisfy the conditions of Theorem 1 [10, p. 241]. 


\section{Special basis in the space of the solutions}

Let functions $w^{1}, \ldots, w^{m} \in F(X)$ be linearly independent. Then the following lemma is valid (see [17], too).

Lemma 3. Functionals $f_{1}, \ldots, f_{m}$ are linearly independent on $\operatorname{span}\left(w^{1}, \ldots\right.$, $\left.w^{m}\right) \subset F(X)$ if and only if $D(\boldsymbol{f})[\boldsymbol{w}] \neq 0$.

Let us consider homogeneous linear differential equation (3.1) and let $\boldsymbol{u}=$ $\left\{u^{1}, \ldots, u^{m}\right\}$ be a fixed basis of the $m$-dimensional linear space $S$ of solutions. We investigate additional conditions:

$$
\left\langle L_{1}, u\right\rangle=0, \ldots,\left\langle L_{m}, u\right\rangle=0, \quad u \in S,
$$

where $L_{1}, \ldots, L_{m} \in S^{*}$ are linearly independent linear functionals. Denote $\boldsymbol{L}=\left(L_{1}, \ldots, L_{m}\right)$. Let us introduce new functions

$$
\hat{v}^{i}[\boldsymbol{u}](x):=D\left(L_{1}, \ldots, L_{i-1}, \delta_{x}, L_{i+1}, \ldots, L_{m}\right)[\boldsymbol{u}], i=\overline{1, m} .
$$

For these functions $\left\langle L_{i}, \hat{v}^{j}[\boldsymbol{u}]\right\rangle=\delta_{i}^{j} D(\boldsymbol{L})[\boldsymbol{u}], i, j=\overline{1, m}$. Thus, we can extend Lemma 3.

Lemma 4. Let $\left\{u^{1}, \ldots, u^{m}\right\}$ be the basis of linear space $S$. Then the next propositions are equivalent:

1. The functionals $L_{1}, \ldots, L_{m}$ are linearly independent;

2. The functions $\hat{v}^{1}, \ldots, \hat{v}^{m}$ are linearly independent;

3. $D(\boldsymbol{L}) \neq 0$.

Remark 5. The condition $D(\boldsymbol{L})=0$ does not depend on the fundamental system. Therefore, we use the notation $D(\boldsymbol{L})$ instead of $D(\boldsymbol{L})[\boldsymbol{u}]$ in this lemma.

The equalities

$$
\begin{aligned}
W[\hat{\boldsymbol{v}}](x) & =W[\boldsymbol{u}](x) \cdot(D(\boldsymbol{L})[\boldsymbol{u}])^{m-1}, \widetilde{W}[\hat{\boldsymbol{v}}](x, y)=\widetilde{W}[\boldsymbol{u}](x, y)(D(\boldsymbol{L})[\boldsymbol{u}])^{m-1}, \\
D[\hat{\boldsymbol{v}}](\boldsymbol{x}) & =D[\boldsymbol{u}](\boldsymbol{x})(D(\boldsymbol{L})[\boldsymbol{u}])^{m-1}
\end{aligned}
$$

are valid, too. So, the propositions in Lemma 4 are equivalent to $W[\hat{\boldsymbol{v}}](x) \neq 0$.

If the functionals $L_{1}, \ldots, L_{m}$ are linearly independent, and

$$
v^{i}(x):=\frac{D\left(L_{1}, \ldots, L_{i-1}, \delta_{x}, L_{i+1}, \ldots, L_{m}\right)[\boldsymbol{u}]}{D(\boldsymbol{L})[\boldsymbol{u}]}=\frac{\hat{v}^{i}[\boldsymbol{u}](x)}{D(\boldsymbol{L})[\boldsymbol{u}]}, \quad i=\overline{1, m},
$$

then two bases $\left\{v^{1}, \ldots, v^{m}\right\}$ and $\left\{L_{1}, \ldots, L_{m}\right\}$ are biorthogonal: $\left\langle L_{i}, v^{j}\right\rangle=\delta_{i}^{j}$, $i, j=\overline{1, m}$, and

$$
\begin{gathered}
D[\boldsymbol{v}](\boldsymbol{x})=\frac{D[\boldsymbol{u}](\boldsymbol{x})}{D(\boldsymbol{L})[\boldsymbol{u}]}, W[\boldsymbol{v}](x)=\frac{W[\boldsymbol{u}](x)}{D(\boldsymbol{L})[\boldsymbol{u}]}, \quad \widetilde{W}[\boldsymbol{v}](x, y)=\frac{\widetilde{W}[\boldsymbol{u}](x, y)}{D(\boldsymbol{L})[\boldsymbol{u}]}, \\
V[\boldsymbol{v}](x, y)=V[\boldsymbol{u}](x, y)=V(x, y), G^{c}[\boldsymbol{v}](x, y)=G^{c}[\boldsymbol{u}](x, y)=G^{c}(x, y) .
\end{gathered}
$$

Remark 6. The Propositions in Lemma 4 are valid, if we take $\left\{v^{1}, \ldots, v^{m}\right\}$ instead of $\left\{\hat{v}^{1}[\boldsymbol{u}], \ldots, \hat{v}^{m}[\boldsymbol{u}]\right\}$. The definition of $\boldsymbol{v}:=\left[v^{1}, \ldots, v^{m}\right]$ is invariant with respect to basis $\left\{u^{1}, \ldots, u^{m}\right\}$. 


\section{Linear differential equation with additional conditions}

In this section, we consider the $m$-order inhomogeneous differential equation

$$
\mathcal{L} u:=u^{(m)}+a^{m-1}(x) u^{(m-1)}+\cdots+a^{1}(x) u^{\prime}+a^{0}(x) u=f(x),
$$

where $f \in C[0, L]$, with $m$ additional conditions

$$
\left\langle L_{i}, u\right\rangle=f_{i} \in \mathbb{K}, \quad i=\overline{1, m}
$$

where $L_{1}, \ldots, L_{m}$ are linearly independent functionals in $C^{m}[0, L]$. We denote $\boldsymbol{L}=\left(L_{1}, \ldots, L_{m}\right), \boldsymbol{f}=\left(f_{1}, \ldots, f_{m}\right), \tilde{\boldsymbol{f}}=[\underbrace{0, \ldots, 0}_{m-1}, f]$.

\subsection{The solution to an inhomogeneous problem with homogeneous additional conditions}

Let $\left\{u^{1}, \ldots, u^{m}\right\}$ be a fixed fundamental system of homogeneous equation (3.1) and $\boldsymbol{u}=\left[u^{1}, \ldots, u^{m}\right]$. Then the general solution of this equation is $u_{h}(x)=$ $\boldsymbol{C u}(x)$, where $\boldsymbol{C}=\left(C_{1}, \ldots, C_{m}\right)$ are arbitrary constants. We replace the constants $\boldsymbol{C}$ with the functions $\boldsymbol{c}(x)=\left(c_{1}(x), \ldots, c_{m}(x)\right)$, respectively (Method of Variation of Parameters, see [3]). Then we substitute $u(x)=\boldsymbol{c}(x) \boldsymbol{u}(x)$ into a inhomogeneous equation (5.1) and determine these functions $\boldsymbol{c}(x)$ [3]. Finally, we obtain:

$$
u(x)=\int_{0}^{L} H(x-s) V(x, s) f(s) d s+\boldsymbol{A u}(x)=\left(G^{c}(x, s), f(s)\right)_{X}+\boldsymbol{A u}(x),
$$

where $(g, f)_{X}:=\int_{0}^{L} g(x) f(x) d x, g \in L^{1}(0, L), f \in C[0, L]$. We use this formula for the special basis $\left\{v^{1}, \ldots, v^{m}\right\}$ (see Eq. (4.3)). In this case, we have

$$
u(x)=\left(G^{c}(x, s), f(s)\right)_{X}+A_{1} v^{1}(x)+\cdots+A_{m} v^{m}(x) .
$$

Let us have homogeneous conditions

$$
\left\langle L_{1}, u\right\rangle=0, \ldots,\left\langle L_{m}, u\right\rangle=0
$$

Then, by substituting general solution (5.4) into homogeneous additional conditions, we find:

$$
A_{i}=-\left\langle L_{i}(y),\left(G^{c}(y, s), f(s)\right)_{X}\right\rangle=-\left(\left\langle L_{i}(y), G^{c}(y, s)\right\rangle, f(s)\right)_{X}, \quad i=\overline{1, m} .
$$

Next we obtain a formula of the solution in the case of the $m$-order linear ODE with $m$ additional homogeneous conditions

$$
u_{f}(x)=\left(\left\langle\delta_{x}(y)-\boldsymbol{L}(y) \boldsymbol{v}(x), G^{c}(y, s)\right\rangle, f(s)\right)_{X} .
$$




\subsection{Inhomogeneous problem}

Let us consider homogeneous equation (3.1) with additional conditions (5.2): $\mathcal{L} u=0,\left\langle L_{i}, u\right\rangle=f_{i}, i=\overline{1, m}$. We can find a solution $u_{h}(x)=\boldsymbol{f} \boldsymbol{v}(x)$ to this problem if the general solution is inserted into additional conditions. The solution of inhomogeneous problems has the form $u(x)=u_{f}(x)+u_{h}(x)$.

Theorem 1. The solution of problem (5.1)-(5.2) can be written by the formula

$$
u(x)=\left(\left\langle\delta_{x}(y)-\boldsymbol{L}(y) \boldsymbol{v}(x), G^{c}(y, s)\right\rangle, f(s)\right)_{X}+\boldsymbol{f} \boldsymbol{v}(x) .
$$

Formula (5.7) is known (in more classical forms) in very different cases of classical boundary conditions of the first, second, and third type. The investigation of linear and quasi-linear problems with NBCs (see, [28, 31]) requires the general theory how to construct a solution with very different boundary conditions. Formula (5.7) can be effectively employed to get the solutions to a linear $m$-order differential equation, where $a^{0}(x), \ldots, a^{m-1}(x)$ are not constants and with any right-hand side function $f(x)$ and any functionals $L_{1}, \ldots$, $L_{m}$ and any $f_{1}, \ldots, f_{m}$, provided that the general solution of the homogeneous equation is known. In this paper, we use it to get formulae for Green's function.

\subsection{Relation between two solutions}

Next, let us consider two problems with the same $m$-order nonhomogeneous differential equation with a differential operator as in the previous subsection:

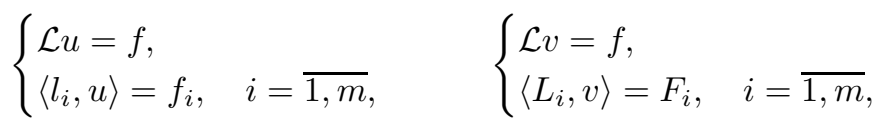

and $D(\boldsymbol{L}) \neq 0$. The difference $w=v-u$ satisfies the problem with a homogeneous equation

$$
\left\{\begin{array}{l}
\mathcal{L} w=0, \\
\left\langle L_{i}, w\right\rangle=F_{i}-\left\langle L_{i}, u\right\rangle, \quad i=\overline{1, m} .
\end{array}\right.
$$

Thus, it follows from formula $(5.7)$ that $w(x)=\boldsymbol{F} \boldsymbol{v}(x)-\langle\boldsymbol{L}(y) \boldsymbol{v}(x), u(y)\rangle$ or

$$
v(x)=u(x)-\langle\boldsymbol{L}(y) \boldsymbol{v}(x), u(y)\rangle+\boldsymbol{F} \boldsymbol{v}(x),
$$

and we can express the solution of the second problem (5.8) via the solution of the first problem. We can rewrite (5.9) as follows

$$
v(x)=\frac{D\left(\boldsymbol{L}, \delta_{x}\right)[\boldsymbol{u}, u]}{D(\boldsymbol{L})[\boldsymbol{u}]}+\boldsymbol{F} \boldsymbol{v}(x) .
$$

Note that, in this formula, the function $u$ is in the first term only and $v(x)$ is invariant with respect to the basis $\left\{u^{1}, \ldots, u^{m}\right\}$. 


\section{Green's functions}

We propose the definition of Green's function (see, $[3,4,23])$. The fundamental Solution $G(x, y)$ of equation (5.1) is Green's function for BVP (5.1)-(5.2) (in the case of local or periodic BC), if it satisfies (as a function of $x$ ) homogeneous boundary conditions (5.2), too. We will use here a slightly more general definition of Green's function.

Definition 3. A GFS $G(x, y)$ of equation (5.1) is Green's function for (5.1)(5.2) if it satisfies (as a function of $x$ ) homogeneous additional conditions (5.2) for $y \in[0, L] \backslash \Xi_{N}$, i.e.,

$$
\left\langle L_{i}(x), G(x, y)\right\rangle=0, \quad i=\overline{1, m}, \quad y \in[0, L] \backslash \Xi_{N} .
$$

If Green's function $G(x, y)$ exists for problem (5.1)-(5.2), then its solution allows the following integral representation:

$$
u(x)=\int_{0}^{L} G(x, y) f(y) d y .
$$

So, we have

$$
\left\langle L_{i}, u\right\rangle=\int_{0}^{L}\left\langle L_{i}(x), G(x, y)\right\rangle f(y) d y=0 .
$$

\subsection{Green's functions for a linear differential equation with addi- tional conditions}

Let us consider an inhomogeneous equation with an operator (5.1): $\mathcal{L}: U \rightarrow F$, where $F=C[0, L]$ and additional homogeneous conditions define the subspace $U=\left\{u \in C^{m}[0, L]:\left\langle L_{1}, u\right\rangle=0, \ldots,\left\langle L_{m}, u\right\rangle=0\right\}$. We derive a formula for the solution (see Theorem 1 for $\boldsymbol{f}=\mathbf{0}$ ):

$$
u(x)=\int_{0}^{L}\left\langle\delta_{x}(y)-\boldsymbol{L}(y) \boldsymbol{v}(x), G^{c}(y, s)\right\rangle f(s) d s .
$$

Thus, we have proved the following lemma about Green's function.

Lemma 5. Green's function for problem (5.1) with $m$ homogeneous additional conditions $\left\langle L_{1}, u\right\rangle=0, \ldots,\left\langle L_{m}, u\right\rangle=0$ is equal to:

$$
G(x, s)=\left\langle\delta_{x}(y)-\boldsymbol{L}(y) \boldsymbol{v}(x), G^{c}(y, s)\right\rangle=\frac{D\left(\boldsymbol{L}, \delta_{x}\right)\left[\boldsymbol{u}, G^{c}(\cdot, s)\right]}{D(\boldsymbol{L})[\boldsymbol{u}]} .
$$

Green's function $G(x, s)=G[\overline{\boldsymbol{u}}](x, s)=G[\boldsymbol{u}](x, s)$, i.e., it is invariant with respect to the basis $\left\{u^{1}, \ldots, u^{m}\right\}$. It is easy to verify that $G^{c}(x, s)$ is Green's function for problem (5.1) with the special additional (in this case, initial conditions) conditions $u(0)=0, \ldots, u^{(m-1)}(0)=0$.

For the theoretical investigation of problems with nonlocal boundary conditions, the next result on the relations between Green's functions $G^{u}(x, s)$ and $G^{v}(x, s)$ of two inhomogeneous problems with the same $f$ :

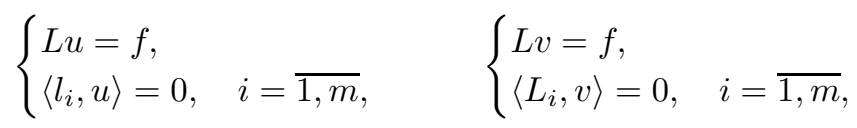


is useful.

Theorem 2. The relations between Green's functions $G^{v}(x, s)$ and $G^{u}(x, s)$ for problems (6.3) are:

$$
G^{v}(x, s)=\left\langle\delta_{x}(y)-\boldsymbol{L}(y) \boldsymbol{v}(x), G^{u}(y, s)\right\rangle=\frac{D\left(\boldsymbol{L}, \delta_{x}\right)\left[\boldsymbol{u}, G^{u}(\cdot, s)\right]}{D(\boldsymbol{L})[\boldsymbol{u}]} .
$$

Proof. The proof of these relations follows from Eq. (5.10) (the case $\boldsymbol{F}=\mathbf{0}$ ) and integral representation (6.1) of the solutions $u$ and $v$.

\section{Applications to problems with NBC}

Let us investigate Green's function for the problem with nonlocal boundary conditions

$$
\begin{aligned}
& \mathcal{L} u:=u^{(m)}+a^{m-1}(x) u^{(m-1)}+\cdots+a^{1}(x) u^{\prime}+a^{0}(x) u=f(x), \\
& \left\langle L_{i}, u\right\rangle:=\left\langle\kappa_{i}, u\right\rangle-\gamma_{i}\left\langle\varkappa_{i}, u\right\rangle=0, i=\overline{1, m}
\end{aligned}
$$

where $a^{i} \in C[0, L], f \in C(0, L)$. We can write many problems with nonlocal boundary conditions (NBC) in this form, where $\left\langle\kappa_{i}, u\right\rangle:=\left\langle\kappa_{i}(x), u(x)\right\rangle$ is a classical part, and $\left\langle\varkappa_{i}, u\right\rangle:=\left\langle\varkappa_{i}(x), u(x)\right\rangle, i=\overline{1, m}$, is a nonlocal part of boundary conditions. For example, the functionals $\varkappa_{i}, i=\overline{1, m}$, can describe the multi-point $\left(\xi_{j} \in[0,1], j=\overline{1, m}\right)$ or integral NBCs

$$
\langle\varkappa, u\rangle=\sum_{j=1}^{m} \sum_{k=1}^{m}\left(\varkappa_{j}^{k} u^{(k-1)}\left(\xi_{j}\right)\right), \quad\langle\varkappa, u\rangle=\int_{0}^{1} \varkappa(t) u(t) d t,
$$

and the functional $\kappa_{i}, i=\overline{1, m}$, can describe the local (classical) boundary conditions.

If $\gamma_{1}, \ldots, \gamma_{m}=0$, then problem (7.1)-(7.2) becomes classical. Suppose that there exists Green's function $G^{\mathrm{cl}}(x, s)$ for this classical case. Then, Green's function exists for problem (7.1)-(7.2) if $\vartheta=D(\boldsymbol{L})[\boldsymbol{u}] \neq 0$. For $L_{i}=\kappa_{i}-\gamma_{i} \varkappa_{i}$, $i=\overline{1, m}$, we derive

$$
\vartheta=\sum_{\sigma_{1}=0, \ldots, \sigma_{m}=0}^{1} \prod_{j=1}^{m}\left(-\gamma_{j}\right)^{\sigma_{j}} D\left(\left(\varkappa_{1}^{\sigma_{1}} \kappa_{1}^{1-\sigma_{1}}\right), \ldots,\left(\varkappa_{m}^{\sigma_{m}} \kappa_{m}^{1-\sigma_{m}}\right)\right) .
$$

If we define the matrices $\mathbf{K}:=\left(\kappa_{i j}\right), \kappa_{i j}=\left\langle\kappa_{j}, u_{i}\right\rangle, \mathbf{N}:=\left(\varkappa_{i j}\right), \varkappa_{i j}=\left\langle\varkappa_{j}, u_{i}\right\rangle$, $\boldsymbol{\Gamma}:=\left(\gamma_{i} \delta_{i j}\right)$, then the condition $\vartheta \neq 0$ is equivalent to $\operatorname{det}\left(\mathbf{I}-\boldsymbol{\Gamma} \mathbf{N K}^{-1}\right) \neq 0$. This result generalizes a similar condition for the second order equation (see, $[1,2,25])$. Since $\left\langle\kappa_{i}(\cdot), G^{\mathrm{cl}}(\cdot, s)\right\rangle=0, i=\overline{1, m}$, we can rewrite formula $(6.4)$ as

$$
G(x, s)=G^{\mathrm{cl}}(x, s)+\sum_{j=1}^{m} \gamma_{j}\left\langle\varkappa_{j}(y), G^{\mathrm{cl}}(y, s)\right\rangle v^{j}(x) .
$$


Example 3. Let us consider the problem

$$
\begin{gathered}
u^{(m)}=f(x), \quad x \in(0,1), \\
u(0)=\beta u(\alpha), \quad u^{\prime}(0)=\cdots=u^{(m-2)}(0)=0, \quad u(1)=\gamma u(\eta),
\end{gathered}
$$

We can take $u^{j}(x)=x^{j-1} /(j-1) !, j=\overline{1, m}$. Then

$$
\widetilde{W}(x, s)=\frac{(x-s)^{m-1}}{(m-1) !}, \quad W(s)=1 .
$$

So, we get

$$
V(x, s)=\frac{(x-s)^{m-1}}{(m-1) !}, \quad G^{c}(x, s)=H(x-s) \frac{(x-s)^{m-1}}{(m-1) !} .
$$

We find $\widetilde{W}(x, s)$ directly, but it is easier to find $V(x, s)$ using properties $(2.4)$ (2.5). For a problem with boundary conditions

$$
u(0)=0, \quad u^{\prime}(0)=\cdots=u^{(m-2)}(0)=0, \quad u(1)=0,
$$

we derive

$$
D(\boldsymbol{L})[\boldsymbol{u}]=\frac{1}{(m-1) !}, \quad D\left(\boldsymbol{L}, \delta_{x}\right)\left[\boldsymbol{u}, G^{c}(\cdot, s)\right]=\frac{G^{c}(x, s)-x^{m-1} V(1, s)}{(m-1) !},
$$

and "classical" Green's function

$$
\begin{aligned}
G^{\mathrm{cl}}(x, s) & =H(x-s) V(x, s)-x^{m-1} V(1, s) \\
& =\frac{1}{(m-1) !} \begin{cases}-x^{m-1}(1-s)^{m-1}, & x \leq s, \\
(x-s)^{m-1}-x^{m-1}(1-s)^{m-1}, & s \leq x .\end{cases}
\end{aligned}
$$

For a "nonlocal" problem with boundary conditions $u(0)-\beta u(\alpha)=0$, $u^{\prime}(0)=\cdots=u^{(m-2)}(0)=0, u(1)-\gamma u(\eta)=0$, we calculate

$$
\begin{aligned}
D(L)[\boldsymbol{u}] & =\left((1-\beta)\left(1-\gamma \eta^{m-1}\right)+(1-\gamma) \beta \alpha^{m-1}\right) /(m-1) !, \\
D\left(\delta_{x}, L_{2}, \ldots, L_{m}\right)[\boldsymbol{u}] & =\left(1-\gamma \eta^{m-1}-(1-\gamma) x^{m-1}\right) /(m-1) ! \\
D\left(L_{1}, \ldots, L_{m-1}, \delta_{x}\right)[\boldsymbol{u}] & =\left((1-\beta) x^{m-1}+\beta \alpha^{m-1}\right) /(m-1) !
\end{aligned}
$$

It follows from Eq. (7.4) that

$$
\begin{aligned}
G(x, s)= & G^{\mathrm{cl}}(x, s)+\beta G^{\mathrm{cl}}(\alpha, s) \frac{1-\gamma \eta^{m-1}-(1-\gamma) x^{m-1}}{(1-\beta)\left(1-\gamma \eta^{m-1}\right)+(1-\gamma) \beta \alpha^{m-1}} \\
& +\gamma G^{\mathrm{cl}}(\eta, s) \frac{(1-\beta) x^{m-1}+\beta \alpha^{m-1}}{(1-\beta)\left(1-\gamma \eta^{m-1}\right)+(1-\gamma) \beta \alpha^{m-1}}
\end{aligned}
$$


if $\vartheta:=(1-\beta)\left(1-\gamma \eta^{m-1}\right)+(1-\gamma) \beta \alpha^{m-1} \neq 0$. Thus,

$$
\begin{aligned}
& G(x, s)=\frac{1}{(m-1) !} \begin{cases}-x^{m-1}(1-s)^{m-1}, & x \leq s, \\
(x-s)^{m-1}-x^{m-1}(1-s)^{m-1}, & s \leq x,\end{cases} \\
& +\beta \frac{1-\gamma \eta^{m-1}-(1-\gamma) x^{m-1}}{(1-\beta)\left(1-\gamma \eta^{m-1}\right)+(1-\gamma) \beta \alpha^{m-1}} \begin{cases}-\frac{\alpha^{m-1}(1-s)^{m-1}}{(m-1) !}, & \alpha \leq s, \\
\frac{(\alpha-s)^{m-1}-\alpha^{m-1}(1-s)^{m-1}}{(m-1) !}, & s \leq \alpha,\end{cases} \\
& +\gamma \frac{(1-\beta) x^{m-1}+\beta \alpha^{m-1}}{(1-\beta)\left(1-\gamma \eta^{m-1}\right)+(1-\gamma) \beta \alpha^{m-1}} \begin{cases}-\frac{\eta^{m-1}(1-s)^{m-1}}{(m-1) !}, & \eta \leq s, \\
\frac{(\eta-s)^{m-1}-\eta^{m-1}(1-s)^{m-1}}{(m-1) !}, & s \leq \eta .\end{cases}
\end{aligned}
$$

Remark 7. The problem (7.5)-(7.6) was considered in the case $\alpha=\eta$ by Ji and Guo [7]. Green's function for this problem is of the form

$$
\begin{aligned}
G(x, s) & =G^{\mathrm{cl}}(x, s)+\frac{x^{m-1}(\gamma-\beta)+\beta}{1-\beta-\eta^{m-1}(\gamma-\beta)} G^{\mathrm{cl}}(\eta, s) \\
& =\frac{1}{(m-1) !} \begin{cases}-x^{m-1}(1-s)^{m-1}, & x \leq s, \\
(x-s)^{m-1}-x^{m-1}(1-s)^{m-1}, & s \leq x,\end{cases} \\
& +\frac{x^{m-1}(\gamma-\beta)+\beta}{1-\beta-\eta^{m-1}(\gamma-\beta)} \begin{cases}-\frac{\eta^{m-1}(1-s)^{m-1}}{(m-1) !}, & \eta \leq s, \\
\frac{(\eta-s)^{m-1}-\eta^{m-1}(1-s)^{m-1}}{(m-1) !}, & s \leq \eta .\end{cases}
\end{aligned}
$$

Remark 8. Problem (7.5)-(7.6) was considered in the case $\beta=0$ by Hao, Liu and $\mathrm{Wu}\left[6\right.$, Section 2]. As $\gamma \eta^{m-1} \neq 1$ Green's function for this problem is equal to

$$
\begin{aligned}
G(x, s) & =G^{\mathrm{cl}}(x, s)+\gamma G^{\mathrm{cl}}(\eta, s) \frac{x^{m-1}}{1-\gamma \eta^{m-1}} \\
& = \begin{cases}\phi(s) x^{m-1} /(m-1) !, & 0 \leq x \leq s \leq 1 \\
\left(\phi(s) x^{m-1}+(x-s)^{m-1}\right) /(m-1) !, & 0 \leq s \leq x \leq 1\end{cases}
\end{aligned}
$$

where

$$
\phi(s)= \begin{cases}-(1-s)^{m-1} /\left(1-\gamma \eta^{m-1}\right), & \eta \leq s \\ -\left((1-s)^{m-1}-\gamma(\eta-s)^{m-1}\right) /\left(1-\gamma \eta^{m-1}\right), & s \leq \eta\end{cases}
$$

\section{Conclusions}

The main result of this paper is that Green's function for the linear ODE with additional conditions is related with Green's function of a similar problem, and this relation is expressed by formulae (6.4). If $D(\boldsymbol{L})[\boldsymbol{u}] \neq 0$, the functionals $L_{1}, \ldots, L_{m}$ are linearly independent. This condition yields the necessary and sufficient condition for the existence of Green's function for the problem with $m$ functional conditions. We give few examples, but formulae (6.4) can be applied to a very wide class of problems with nonconstant coefficients and various boundary conditions as well as additional conditions. 


\section{References}

[1] R. Čiegis, A. Štikonas, O. Štikonienè and O. Suboč. A monotonic finitedifference scheme for a parabolic problem with nonlocal conditions. Differ. Equ., 38(7):1027-1037, 2002. Doi:10.1023/A:1021167932414.

[2] R. Čiegis, A. Štikonas, O. Štikonienė and O. Suboč. Stationary problems with nonlocal boundary conditions. Math. Model. Anal., 6(2):178-191, 2001.

[3] E.A. Coddington and N. Levison. Theory of Ordinary Differential Equations. McGraw Hill Book Co., Inc., New York, Toronto, London, 1955.

[4] D.G. Duffy. Green's functions with Applications. Chapman \& Hall/CRC Press, 2001.

[5] J.R. Graef, L. Kong and B. Yang. Positive solutions of a nonlinear higher order boundary-value problem. Discrete and Continuous Dynamical Systems, pp. 276$285,2009$.

[6] X. Hao, L. Liu and Y. Wu. Positive solutions for nonlinear $n$ th-order singular nonlocal boundary value problems. Bound. Value Probl., 2007:1-10, 2007. Doi:10.1155/2007/74517.

[7] Y. Ji and Y. Guo. The existence of countably many positive solutions for nonlinear $n$ th-order three-point boundary value problems. Bound. Value Probl., 2009:1-18, 2009. Doi:10.1155/2009/572512.

[8] W. Jiang. Positivie solutions for a high-order multi-point boundary-value problem in banach spaces. Electron. J. Differential Equations, 2008(70):1-11, 2008.

[9] E. Kamke. Differentialgleichungen: Losungsmethoden und Losungen, I, Gewohnliche Differentialgleichungen. B. G. Teubner, Leipzig, 1977.

[10] A.N. Kolmogorov and S.V. Fomin. Introductory Real Analysis. Dover Publications, Inc., New York, 2000. (Revised English Edition)

[11] A.I. Kostrikin. Introduction to Algebra. Berlin: Springer-Verlag, 1982.

[12] Y. Liu and D. O'Regan. Multiplicity results using bifurcation techniques for a class of fourth-order $m$-point boundary value problems. Bound. Value Probl., 2009:1-20, 2009. Doi:10.1155/2009/970135.

[13] S.K. Ntouyas. Nonlocal initial and boundary value problems: a survey. In A. Cañada, P. Drábek and A. Fonda(Eds.), Handbook of Differential Equations, volume 2 of Ordinary Differential Equations, pp. 461-558, North Holland, 2005. Elsevier.

[14] S. Pečiulytè and A. Štikonas. On positive eigenfunctions of Sturm-Liouville problem with nonlocal two-point boundary condition. Math. Model. Anal., 12(2):215226, 2007. Doi:10.3846/1392-6292.2007.12.215-226.

[15] A.D. Polyanin. Handbook of Linear Partial Differential Equations for Engineers and Scientists. Chapman \& Hall/CRC Press, Boca Raton, 2002.

[16] S. Roman and A. Štikonas. Green's functions for stationary problems with nonlocal boundary conditions. Lith. Math. J., 49(2):190-202, 2009. Doi:10.1007/s10986-009-9041-0.

[17] S. Roman and A. Štikonas. Third-order linear differential equation with three additional conditions and formula for Green's function. Lith. Math. J., 50(4):426446, 2010. Doi:10.1007/s10986-010-9097-x. 
[18] S. Roman and A. Štikonas. Green's functions for stationary problems with four-point nonlocal boundary conditions. In V. Kleiza, S. Rutkauskas and A. Štikonas(Eds.), Differential Equations and Their Applications (DETA'2009), pp. 123-130. Kaunas University of Technology, 2009.

[19] S. Roman and A. Štikonas. Green's function for discrete second-order problems with nonlocal boundary conditions. Boundary Value Problems, 2011(Article ID 767024):1-23, 2011. Doi:10.1155/2011/767024.

[20] M. Sapagovas, G. Kairyte, O. Štikonienė and A. Štikonas. Alternating direction method for a two-dimensional parabolic equation with a nonlocal boundary condition. Math. Model. Anal., 12(1):131-142, 2007. Doi:10.3846/1392-6292.2007.12.131-142.

[21] M.P. Sapagovas and A.D. Stikonas. On the structure of the spectrum of a differential operator with a nonlocal condition. Differ. Equ., 41(7):1010-1018, 2005. Doi:10.1007/s10625-005-0242-y.

[22] A. Skučaitè, K. Skučaitė-Bingelè, S. Pečiulytė, and A. Štikonas. Investigation of the spectrum for the Sturm-Liouville problem with one integral boundary condition. Nonlinear Anal., Model. Control, 15(4):501-512, 2010.

[23] I. Stakgold and M. Holst. Green's Functions and Boundary Value Problems. Wiley-Interscience, Hoboken, New Jersey, 2010.

[24] A. Štikonas. The Sturm-Liouville problem with a nonlocal boundary condition. Lith. Math. J., 47(3):336-351, 2007. Doi:10.1007/s10986-007-0023-9.

[25] A. Štikonas. Investigation of characteristic curve for Sturm-Liouville problem with nonlocal boundary conditions on torus. Math. Model. Anal., 16(1):1-229, 2011. Doi:10.3846/13926292.2011.552260.

[26] A. Štikonas and S. Roman. Stationary problems with two additional conditions and formulae for Green's functions. Numer. Funct. Anal. Optim., 30(9):11251144, 2009. Doi:10.1080/01630560903420932.

[27] A. Štikonas and O. Štikonienè. Characteristic functions for Sturm-Liouville problems with nonlocal boundary conditions. Math. Model. Anal., 14(2):229246, 2009. Doi:10.3846/1392-6292.2009.14.229-246.

[28] J.-P. Sun and H.-E. Zhang. Existence of solutions to third-order $m$-point boundary-value problems. Electron. J. Differ. Eqns., 2008(125):1-9, 2008.

[29] D. Xie, Y. Liu and C. Bai. Green's function and positive solutions of a singular $n$ th-order three-point boundary value problem on time scales. Electron. J. Differ. Eqns., 2009(38):1-14, 2009.

[30] J. Xu and Z. Wei. Positive solutions for multipoint boundary-value problem with parameters. Electron. J. Differ. Eqns., 2008(106):1-8, 2008.

[31] B. Yang. Positive solutions of a third-order three-point boundary-value problem. Electron. J. Differ. Eqns., 2008(99):1-10, 2008.

[32] Z. Zhao. Positive solutions for singular three-point boundary-value problems. Electron. J. Differ. Eqns., 2007:1-8, 2007. 